\title{
ANXIETY, DEPRESSION OF PREGNANT WOMEN DURING COVID-19 PANDEMIC: ARTICLE REVIEW
}

\author{
Varvara O. Anikina ${ }^{1}$, Svetlana S. Savenysheva ${ }^{1}$, \& Mariia E. Blokh ${ }^{1,2}$ \\ ${ }^{1}$ Saint-Petersburg State University, Saint-Petersburg (Russia) \\ ${ }^{2} \mathrm{Dr}$ D. O. Ott Research Institute of Obstetrics, Gynecology, and Reproduction, Saint-Petersburg (Russia)
}

\begin{abstract}
The article is the review of the available research papers on anxiety, depression, stress and signs of PTSD in pregnant women during the COVID-19 pandemic. Articles were searched in the databases of Scopus, Web of Science, EBSCO, APA using the keywords "pregnancy", "COVID-19", "anxiety"," depression"," stress"," PTSD". For this article review we selected only those research studies that have comparatively large samples, with the most widely used measures: State and Trait Anxiety Inventory (STAI), Generalized Anxiety Disorder (GAD-7), Edinburg Postpartum Depression Scale (EPDS), and Impact of a Traumatic Event Scale (IES-R). In these studies levels of anxiety, depression and PTSD are either compared to the existing cut-off scores for these disorders in the literature or in COVID-19 and pre-COVID cohorts of pregnant women. Some papers include not only women during pregnancy but also postpartum. Data here are presented only on pregnancy. The results show that $22 \%$ to $68 \%$ of pregnant women experience moderate to severe anxiety, and it is two to five times more than the prevalence of anxiety in the literature. The state anxiety has increased more compared to trait anxiety. $14.9 \%-34.2 \%$ of women report on clinically significant levels of depression, and it is twice higher than the pre-existing data. About $10.3 \%$ of pregnant population have PTSD signs which falls into a moderate range. The levels of anxiety, depression and PTSD are significantly higher in COVID-19 cohorts than in pre-COVID samples. The most predicting factor for anxiety, depression and PTSD is the pre-existing mental health disorder of anxiety or depression.
\end{abstract}

Keywords: COVID-19, pregnancy, anxiety, depression, PTSD.

\section{Introduction}

Pregnant women being a vulnerable group of the population, during the COVID-19 pandemic are exposed to a huge number of stress factors - a decrease in the number of planned prenatal visits, the closure of delivery hospitals, self-isolation, lack of physical activity, financial problems, fear of infection, both of their own and their loved ones and the unborn child, as well as the vertical transmission and during breastfeeding. All this can lead to anxiety, depression, distress, which increases the likelihood of complications during pregnancy and delivery outcomes.

Accumulated data from the previous scientific studies show that stress during pregnancy, along with anxiety and depression, can increase vulnerability to infectious diseases (Christian et al., 2010), lead to pregnancy complications (Rubertsson et al., 2014), and increase the likelihood of premature birth (Staneva et al., 2015). Prenatal stress can have a negative impact on the quality of mother-child interaction (Spinelli et al., 2013), contribute to disorders of the child's mental health both after childbirth and later, in preschool and school years (Savenysheva, 2018).

\section{Anxiety and depression of pregnant women during the pandemic}

One of the first studies of the emotional states of pregnant women during the pandemic was in Italy (Saccone et al., 2020). Anxiety was assessed using Spielberger State and Trait Anxiety Inventory (STAI) and signs of PTSD using the Impact of a Traumatic Event Scale (IES-R). The study included 100 women, $48 \%$ in the third trimester. The results showed that $68 \%$ of women demonstrate moderate to severe anxiety, and 53\% report the impact of the pandemic as strong. In another Italian study (Mappa et al., 2020) with 178 pregnant women (mean age 33 years, $61 \%$ in the second trimester), clinically 
significant anxiety levels measured with STAI were reported by $38.2 \%$ of women; the median value for state anxiety was 12 points significantly higher $(\mathrm{p} \leq 0.0001)$ than the median value for trait anxiety.

Another method for assessing the level of anxiety is the Generalized Anxiety Disorder Questionnaire (GAD-7), which has also been used to assess the level of anxiety in pregnant women. In particular, in an American study including 788 pregnant women, moderate symptoms of anxiety (scores from 10 to 14 ) were observed in $21.6 \%$ of pregnant women, and $21.7 \%$ reported on serious symptoms (scores $\geq 15$ ). This means that about half of the sample experience moderate-to-severe anxiety during the pandemic (Preis et al., 2020).

Greek study of 269 pregnant women (27.5\% over 35 years of age) during a 6-week quarantine shows that $27.5 \%$ experience moderate and $10 \%$ strong/very strong state and $10.8 \%$ - moderate and $2.2 \%$ strong/very strong trait anxiety. State anxiety was significantly associated with postpartum depression measured using the Edinburgh Postpartum Depression Scale (EPDS), where 14.9\% of the sample showing signs of severe (scores above 13) postpartum depression (Dagklis et al., 2020).

One of the most comprehensive study is the research conducted by the Belgian authors. It included 2,421 pregnant. The results showed that $69.5 \%$ of pregnant women show signs of postpartum depression as measured by EPDS (scores above 10), with extremely high values (above 13 points) observed in $25.3 \%$ of pregnant women (Ceulemans et al., 2020).

In an Italian study including 389 of whom were pregnant (mean age $32.9 \pm 4.3$ years) using STAI and EPDS the results showed that $64.0 \%$ of pregnant women had clinically high scores for state anxiety, and $44.0 \%$ - for trait anxiety. Moreover, $34.2 \%$ of pregnant women had clinically high scores (more than 13) on EPDS (Molgora et al. 2020).

The study conducted in the United States, involved 1061 women (average age 33.10 \pm 3.77 years, $89.9 \%$ of the white population, $30.7 \%$ - in the third trimester, $45.8 \%$ - after the birth of a child). Anxiety, depression, and signs of PTSD were evaluated in a combined sample of pregnant and postpartum women. The results showed that $36.4 \%$ of the entire sample had clinically significant indicators of depression, $22.7 \%$ - anxiety, $10.3 \%$ showed signs of PTSD; $18.2 \%$ of women show moderate to severe health-related anxiety. At the same time, it was found that before pregnancy in the study sample, $17.5 \%$ of women were diagnosed with depression, $24.5 \%$ with generalized anxiety, and $4.1 \%$ were diagnosed with PTSD. The results of this study revealed that pre-pregnancy depression was a significant predictor of clinically severe depression at the time of the study $(\mathrm{p}<0.01)$; similarly, a diagnosis of PTSD predicted the presence of signs of PTSD during the pandemic $(p<0.01)$. Pre-pregnancy anxiety and health-related anxiety during the pandemic were significant predictors of both anxiety, depression $(\mathrm{p}<0.001)$, and severe signs of PTSD $(\mathrm{p}<0.05)$ at the time of the study (Liu et al., 2021).

\section{Anxiety, depression and PTSD signs in COVID-19 and pre-COVID cohorts of pregnant women}

Some scientific papers present studies comparing samples of pregnant women recruited during the pandemic and before it has begun. One of the first such studies is the work carried out in China (Wu et al., 2020). It involved 4,124 women (average age 30 years, 14\% over 35 years), of whom 2,839 were participants in the study before the outbreak of the pandemic was announced. The researchers used the Edinburgh Postpartum Depression Scale (EPDS). The results show that the average EPDS score in the group during the pandemic period is below the critical threshold of 10 points, but higher than in the group before the pandemic. The same tendency is observed for the anxiety subscales in the EPDS scale. Moreover, there was a significant increase in anxiety and depression due to an increase in the number of reported cases of deaths $(\mathrm{p}<0.005)$. Similar data were obtained in a number of other studies (Dagklis et al., 2020., Kotabagi et al., 2020).

Another study was conducted in Canada and aimed to assess prenatal stress, anxiety, depression, signs of PTSD, and also had questions about mental health disorders. The results were analyzed for two cohorts of women (mean age $29.27 \pm 4.23$ years, gestational age $24.80 \pm 9.42$ weeks), whose data were collected in the period before the COVID-19 pandemic was declared $(n=496)$ and during self-isolation period $(n=1258)$. The results show that pregnant women in the COVID-19 cohort reported higher levels of prenatal stress $(p<0.001)$ compared to pregnant women in the pre-COVID-19 cohort, even when controlling for the woman's age, gestational age, level of education and family income, and the presence of mental health disorders. It was also found that compared to the group before the pandemic, pregnant women from the COVID-19 group were more likely to have more severe and clinically significant symptoms of depression and anxiety $(6.0 \%$ and $10.9 \%$, respectively, $p=0.002)$, more symptoms of PTSD, particularly dissociation $(\mathrm{p}<0.001)$. The data also showed that the presence of a mental health disorder is a significant predictor of anxiety, dissociative symptoms, and mood change (Berthelot et al., 2020). 


\section{Discussion}

Thus, the data from scientific studies obtained in 2020 in different countries and on large samples indicate that from $22 \%$ to $68 \%$ of pregnant women experience moderate to severe anxiety during the pandemic. At the same time cut-off scores presented in the scientific literature in the pre-pandemic time indicate the prevalence of anxiety is in $10-15 \%$ of cases. This means that the levels of anxiety during the pandemic are two to five times higher compared to the average number of the usual prevalence. Some studies indicate the increase of anxiety levels with the increasing numbers of the reported deaths in the mass-media. Other results indicate that state anxiety had e tendency to increase more than trait anxiety, which altogether can be viewed as an adaptive reaction to stressful situation, especially high levels of uncertainty and information presented in mass media. This data should be more observed in the future for the long-term consequences of anxiety, and if state anxiety may get internalized into traits or posttraumatic symptoms in pregnant women and postpartum.

Another result that we obtained from the articles review is that different studies report that $14.9 \%-36.4 \%$ of pregnant and newly mothers experience depression, specifically clinically severe symptoms of postpartum depression measured by EPDS. The cut-off scores presented in the literature demonstrate the prevalence of depression in $10-22 \%$ of cases. This means that about two times more pregnant women experience depression during the pandemic. It should be noted than in some studies high risk of postpartum depression is evaluated at the score of 10 , but most studies use the critical level of 13, and still the number of clinically significant levels of depression are extremely high.

Signs of stress, including clinical levels of PTSD, are noted in $10.3 \%$ of pregnant women during the COVID-19 times, and this is higher than the levels obtained before the pandemic, indicating the presence of signs of PTSD in 5\% of women (up to $18 \%$ - in high-risk groups). This means that pregnant women fall into a moderate range with regard to PTSD signs. But since the reviewed studies were conducted at the beginning of pandemic, it should be observed in more detail if PTSD symptoms sustains, decrease or, possibly we may observe increase in posttraumatic symptoms in pregnant women and after the delivery. It should also be noted that one of the main predicting factors for greater levels of anxiety, depression and PTSD is the pre-existing mental health disorder before pregnancy. This groups of women should be paid special attention to.

\section{Conclusion}

Anxiety, depression and stress levels of pregnant women had greatly increased during the COVID-19 pandemic compared to the pre-pandemic cohorts. This may have further negative consequences not only on women's mental health but also have a great impact on the fetus, delivery outcomes as well derail future child overall development. Scientists and practitioners should continue studying pregnant women and postpartum paying special attention to mental health issues, both of mother and the child, their interaction and other long-term consequences of COVID-19 pandemic on maternal and child mental and physical health. Special attention should be paid to those women with the pre-existing mental health disorders.

\section{Acknowledgements}

Research is supported by RFBR project No. 20-04-60386.

\section{References}

Berthelot N., Lemieux R., Garon-Bissonnette J., Drouin-Maziade C., Martel É., \& Maziade M. (2020). Uptrend in distress and psychiatric symptomatology in pregnant women during the coronavirus disease 2019 pandemic. Acta Obstetricia and Ginecologica Scandinavia, Vol. 99, P. 848-855. https://doi.org/10.1111/aogs.13925

Ceulemans M., Hompes T., Foulon V. (2020). Mental health status of pregnant and breastfeeding women during the COVID-19 pandemic: A call for action. International Journal of Gynecology \& Obstetrics. Vol. 151. №1. DOI: 10.1002/ijgo.13295

Christian L.M., Franco A., Iams J.D., Sheridan J., Glaser R. (2010). Depressive symptoms predict exaggerated inflammatory responses to an in vivo immune challenge among pregnant women. Brain, Behavior and Immunity, Vol. 24, № 1, P. 49-53. DOI: 10.1016/j.bbi.2009.05.055 
Dagklis, T., Tsakiridis I., Mamopoulos A., Athanasiadis A., Pearson R., \& Papazisis G. (2020). Impact of the COVID-19 lockdown on antenatal mental health in Greece. Psychiatry and Clinical Neurosciences, Vol. 74, P. 602-631. DOI:10.1111/pcn.13135

Kotabagi P., Fortune L., Essien S., Nauta M., \& Yoong W. (2020). Anxiety and depression levels among pregnant women with COVID-19. Acta Obstetricia et Gynecologica Scandinavica, Vol. 99, P. 953-954. DOI: 10.1111/aogs.13928

Liu C., Erdei C., Mittal L. (2021) Risk factors for depression, anxiety, and PTSD symptoms in perinatal women during the COVID-19 Pandemic. Psychiatry Research. 2021. Vol. 295 https://doi.org/10.1016/j.psychres.2020.113552

Mappa I., Distefano F., \& Rizzo G. (2020). Effects of coronavirus 19 pandemic on maternal anxiety during pregnancy: a prospectic observational study. Journal of Perinatal Medicine, Vol. 48, № 6, P. 545-550 https://doi.org/10.1515/jpm-2020-0182

Molgora S., Accordini M. (2020) Motherhood in the Time of Coronavirus: The Impact of the Pandemic Emergency on Expectant and Postpartum Women's Psychological Well-Being. Frontiers in Psychology. DOI: 10.3389/fpsyg.2020.567155.

Preis H., Mahaffey B., Heiselman C., \& Lobel M. (2020). Pandemic-related pregnancy stress and anxiety among women pregnant during the COVID-19 pandemic. American Journal of Obstetrics \& Gynecology MFM. DOI: 10.1016/j.ajogmf.2020.100155.

Rubertsson C., Hellstr m J., Cross M., \& Sydsj G. (2014). Anxiety in early pregnancy: prevalence and contributing factors. Archives of Women's Mental Health, Vol. 17, N 3, P. 221-229. DOI: $10.1007 / \mathrm{s} 00737-013-0409-0$

Saccone G., Florio A., Aiello F., Venturella R., De Angelis M.C., Locci M., Bifulco G., Zullo F., \& Di Spiezio Sardo A. (2020). Psychological Impact of COVID-19 in pregnant women. American Journal of Obstetrics and Gynecology, Vol. 223, №2, P. 293-295. DOI: 10.1016/j.ajog.2020.05.003

Savenysheva S.S. (2018). Vliyanie sostoyaniya i otnosheniya k rebenku materi v period beremennosti na posleduyushchee psihicheskoe razvitie rebenka: analiz zarubezhnyh issledovanij [Influence of the state and attitude towards the child of the mother during pregnancy on the subsequent mental development of the child: analysis of foreign studies]. Internet-zhurnal «Mir nauki - Internet journal "World of Science», № 1. https://mir-nauki.com/PDF/14PSMN118.pdf. (In Russ.).

Spinelli M., Poehlmann J., \& Bolt D. (2013). Predictors of parenting stress trajectories in premature infant-mother dyads. The Journal of Family Psychology, Vol. 27, №6, P. 873-883. DOI: $10.1037 / \mathrm{a} 0034652$

Staneva A., Bogossian F., Pritchard M., Wittkowski A. (2015). The effects of maternal depression, anxiety, and perceived stress during pregnancy on preterm birth: A systematic review. Women Birth, Vol. 28, № 3, P. 179-93.

Wu Y., Zhang C., Liu H., Duan C., Li C., Fan J., Li H., Chen L., Xu H., Li X., Guo Y., Wang Y., Li X., Li J., Zhang T., You Y., Li H., Yang S., Tao X., Xu Y., Lao H., Wen M., Zhou Y., Wang J., Chen Y., Meng D., Zhai J., Ye Y., Zhong Q., Yang X., Zhang D., Zhang J., Wu X., Chen W., Dennis C-L., \& Huang H. (2020). Perinatal depressive and anxiety symptoms of pregnant women during the coronavirus disease 2019 outbreak in China. American Journal of Obstetrics and Gynecology, Vol. 223, P. 240e1-240e9. https://doi.org/10.1016/j.ajog.2020.05.009 